\title{
Role of S-100 Immunostain as An Auxiliary Diagnostic Aid in Leprosy
}

\author{
Ramesh Dhakhwa, ${ }^{1}$ Sneh Acharya, ${ }^{1}$ Sailesh Pradhan, ${ }^{1}$ Sanju Babu Shrestha, ${ }^{2}$ Tomoo Itoh ${ }^{2}$ \\ 'Department of Pathology, Kathmandu Medical College, Sinamangal, Kathmandu, Nepal, ${ }^{2}$ Department of Dermatology and \\ Sexually Transmitted Infections, Kathmandu Medical College, Sinamangal, Kathmandu, Nepal, ${ }^{3}$ Department of Diagnostic \\ Pathology, Kobe University Graduate School of Medicine, Kobe, Japan.
}

\section{ABSTRACT}

Introduction: Histopathologic diagnosis of leprosy is difficult when Bacillary Index (BI) is zero and neural involvement are not easily identifiable on routine Hematoxylin and Eosin stain. This study was undertaken to study the role of S-100 immunostaining in demonstrating different patterns of nerve involvement in various types of leprosy.

Methods: Thirty one skin biopsies with clinico-histopathologic diagnoses of leprosy over a period of two years were included in the study. Ten cases of non-lepromatous granulomatous dermatoses (including eight cases of lupus vulgaris and two cases of erythema nodosum) were used as controls. Tissue sections from all cases and controls were stained with Hematoxylin and Eosin (H\&E) stain, Fite stain and S-100 immunostain. The H\&E stained slides were used to study the histopathological features, Fite stained slides for Bacillary Index and S-100 for nerve changes.

Results: Neural changes could be demonstrated in the entire spectrum of leprosy using S-100 immunostaining. The most common pattern of nerve destruction in the tuberculoid spectrum was fragmented and infiltrated whereas lepromatous spectrum showed mostly fragmented nerve twigs. Intact nerves were not detected in any of the leprosy cases.

Conclusions: S-100 immunostain is a useful auxiliary aid to the routine H\&E stain in the diagnosis of leprosy especially tuberculoid spectrum and intermediate leprosy.

Keywords: bacillary index; leprosy; S-100 immunostain.

\section{INTRODUCTION}

Diagnosis of leprosy is based on classical clinical signs, characteristic histopathological findings and demonstration of acid-fast bacilli on skin biopsies. ${ }^{1}$ The diagnosis of lepromatous leprosy is relatively easy with demonstration of bacilli. ${ }^{2}$

In tuberculoid and indeterminate forms, detection rate of Mycobacterium leprae bacilli is however low. ${ }^{3}$ In such cases, histological evidence of active nerve destruction by granulomatous inflammation is accepted as diagnostic of tuberculoid leprosy as nerve damage and sensory impairment remain key factors in the pathogenesis of leprosy. ${ }^{4}$
However, granulomas and inflammatory cells often totally blot out the nerve structure, and identification of nerve remnants in Hematoxylin and Eosin (H\&E) sections becomes difficult. ${ }^{5}$ Recently immunostaining with S-100 has been used for demonstration of neural changes. ${ }^{2,6}$ We carried out this study to find out the role of $\mathrm{S}-100$ immunostaining in demonstrating nerve changes in the entire spectrum of leprosy which would aid in the histopathologic diagnosis of leprosy.

Correspondence: Dr. Ramesh Dhakhwa, Department of Pathology, Kathmandu Medical College, Sinamangal, Kathmandu, Nepal. Email: rdhakhwa@gmail.com, Phone: +9779841287989. 


\section{METHODS}

A prospective descriptive study was carried out in the department of Pathology, Kathmandu Medical College including 31 skin biopsies over a period of two years (October 2014 to September 2016). Ethical approval was taken. The cases were diagnosed as various spectrum of leprosy based of clinico-histopathologic features. Ten cases of non-lepromatous granulomatous dermatoses (including 8 cases of lupus vulgaris and two cases of erythema nodosum) were used as controls. Tissue sections from all cases and controls were stained with H\&E stain, Fite stain and S-100 immunostain. The H\&E stained slides were used to study the histopathological features. Fite stained slides were used to determine Bacillary Index (BI) as per the Ridley's logarithmic scale. ${ }^{7,8}$ For immunostaining with S-100, deparaffinised tissue sections were incubated with S-100 primary antibody (Polyclonal Rabbit Anti S-100, DAKO, Denmark) for one hour followed by blocking with Dako REAL ${ }^{\text {TM }}$ Peroxidase-Blocking solution. The sections were then washed and incubated with secondary antibody for 30 minutes. After proper washing the sections were subjected to $D A B$ colorimetric reaction. Harris Hematoxylin was used for counterstaining. The sections were then studied for nerve changes. Four patterns of nerve alteration were assigned (based on Gupta et al) ${ }^{3}$ : (1) Infiltrated: dark staining, fibrillar structures in a wavy pattern associated with inflammatory cells. (2) Fragmented: small, dark staining structures within granuloma; identified as nerve fragments because of their fibrillar and wavy appearance though no intact nerve could be visualized. (3) Absent: No dark staining fibrillar structures within or outside the granuloma in an adequate biopsy containing subcutaneous fat and/ or multiple granulomas. The absence of detectable nerves in such a section indicated destruction of nerves beyond recognition by the granulomas. (4) Intact only: dark staining, large fibrillar structures in a wavy pattern with no inflammatory cell inside.

The clinical diagnoses, histopathological features and S-100 staining patterns were correlated. Diagnostic efficacy of the test in terms of sensitivity was calculated considering clinico-histopathologic findings as the "gold standard".

\section{RESULTS}

Among the 31 cases, the distribution of various spectra of leprosy is shown in Table 1. Three cases of Lepromatous leprosy showed epidermal flattening. No epidermal changes were observed in other cases of leprosy. Well-formed perivascular and periadnexal granulomas with a dense lymphocytic infiltrate were detected in BT and TT leprosy. One case of indeterminate leprosy showed perineural and periadnexal lymphocytic infiltrate on H\&E sections while another case revealed mild perivascular and periadnexal lymphocytic and macrophage accumulation. Nerves were demonstrable in 17 cases on H\&E staining. Control group showed intact nerves in all ten cases.

\begin{tabular}{|ll|}
\hline $\begin{array}{l}\text { Table 1. Frequency distribution of various spectra of } \\
\text { leprosy. }\end{array}$ \\
\hline Histopathologic Type & $\mathrm{n}(\%)$ \\
Lepromatous leprosy (LL) & $7(22.58)$ \\
Borderline Lepromatous (BL) & $4(12.9)$ \\
Borderline Tuberculoid (BT) & $13(41.94)$ \\
Tuberculoid leprosy (TT) & $5(16.13)$ \\
Indeterminate leprosy & $2(6.45)$ \\
Total & $31(100)$ \\
\hline
\end{tabular}

All skin biopsies were examined exhaustively on Fite stain for lepra bacilli. Bacilli were readily detected on lepromatous spectrum of leprosy with $\mathrm{BI}$ ranging from 3 to 5 whereas no bacilli were seen in the tuberculoid spectrum $(\mathrm{BI}: 0)$. In the two cases of indeterminate leprosy, bacilli were not detected. However, a presumptive diagnosis of indeterminate leprosy was made based on clinical history and histopathologic findings. All the controls were negative for Lepra bacilli.

With the use of S-100 immunostain, neural component was detectable with much clarity than H\&E stain. In two cases of tuberculoid leprosy however nerves were completely destroyed by granulomas. Hence S-100 positivity could not be demonstrated (Table 2 ). The most common pattern of nerve destruction in the tuberculoid spectrum was fragmented and infiltrated whereas the lepromatous spectrum showed mostly fragmented nerve twigs. Intact nerves were not detected in any of the leprosy cases (Table 3). Nerves with sharp margins could be detected in all ten control biopsies but were uninvolved by endoneurial inflammations.

\begin{tabular}{|c|c|c|c|c|c|c|c|}
\hline $\begin{array}{l}\text { Type of } \\
\text { leprosy }\end{array}$ & $\begin{array}{l}\text { Number } \\
\text { of cases }\end{array}$ & $\begin{array}{l}\text { H\&E and } \\
\text { S-100 positive }\end{array}$ & $\begin{array}{l}\text { H\&E Negative and } \\
\text { S-100 positive }\end{array}$ & $\begin{array}{l}\text { H\&E positive and } \\
\text { S-100 negative }\end{array}$ & $\begin{array}{l}\text { H\&E and } \\
\text { S-100 negative }\end{array}$ & $\begin{array}{l}\text { Sensitivity } \\
\text { of } H \& E\end{array}$ & $\begin{array}{l}\text { Sensitivity } \\
\text { of S100 }\end{array}$ \\
\hline LL & 7 & 3 & 4 & - & - & $63.6 \%$ & $100 \%$ \\
\hline $\mathrm{BL}$ & 4 & 1 & 3 & - & - & $57.1 \%$ & $100 \%$ \\
\hline BT & 13 & 10 & 3 & - & - & $81 \%$ & $100 \%$ \\
\hline TL & 5 & 2 & 1 & - & 2 & $75 \%$ & $100 \%$ \\
\hline IL & 2 & 1 & 1 & - & - & $66 \%$ & $100 \%$ \\
\hline
\end{tabular}


Dhakhwa et al. Role of S-100 Immunostain as An Auxiliary Diagnostic Aid in Leprosy

\begin{tabular}{|c|c|c|c|c|c|}
\hline Type of leprosy & Infiltrated & Fragmented & Absent & Intact & Total \\
\hline LL & $1(14.3 \%)$ & $6(85.7 \%)$ & - & - & 7 \\
\hline BL & $1(25 \%)$ & $3(75 \%)$ & - & - & 4 \\
\hline BT & $6(46.15 \%)$ & $7(53.85 \%)$ & - & - & 13 \\
\hline TT & $3(60 \%)$ & - & $2(40 \%)$ & - & 5 \\
\hline Indeterminate leprosy & $1(50 \%)$ & $1(50 \%)$ & - & - & 2 \\
\hline
\end{tabular}

\section{DISCUSSION}

Leprosy is a chronic infectious disease caused by $M$. leprae and predominantly affects skin and peripheral nerves. The disease is endemic in many tropical and subtropical countries. The Indian subcontinent, Southeast Asia, sub-Saharan countries in Africa and Brazil comprise the most affected areas. ${ }^{9-11}$

Leprosy is diagnosed based on clinical presentation, histomorphologic features using $\mathrm{H} \& \mathrm{E}$ stain and $\mathrm{BI}$ using Fite stain. The diagnosis of lepromatous leprosy is fairly straightforward with demonstration of bacilli but the diagnosis of tuberculoid leprosy and indeterminate forms are relatively difficult on routine H\&E staining. ${ }^{2}$ We had no difficulty in providing a confident diagnosis of lepromatous spectrum of leprosy as all of the cases had $\mathrm{BI}$ of 3 or higher with characteristic histopathologic findings. However, for the tuberculoid spectra, bacilli could not be detected even after rigorous search. In such cases, the presence of inflammatory cells or granulomas in the endoneurium is an important indicator of leprosy. ${ }^{12}$ Nerve remnants within granulomas are however confused with epithelioid cells, fragments of smooth muscle or endothelial cells. ${ }^{5} \mathrm{~S}-100$ is an immunohistochemical marker for Schwann cells, melanocytes, Langerhans cells, chondrocytes, and myoepithelial cells. ${ }^{4}$ Previous studies have demonstrated S-100 as an effective aid for the diagnosis of leprosy especially the tuberculoid spectrum. ${ }^{5,12-14}$ Thomas et al have found that S-100 is a sensitive and reliable marker of nerve damage. The positive staining of Schwann cells allows easy recognition of intact dermal nerve twigs or their fragments. ${ }^{4,15}$ Gupta et al. reported four patterns of nerve damage demonstrable on S-100 immunostaining, namely; Infiltrated, Fragmented, Absent and Intact. ${ }^{3}$

In the present study, H\&E staining alone could detect neural inflammation or destruction in 17 cases. But when S-100 stain was applied, the nerve fragments were readily identifiable in 29 cases. In two of the cases of tuberculoid leprosy, nerves could not be detected even with $\mathrm{S}-100$ staining and $\mathrm{BI}$ was $\mathrm{O}$ as well on Fite staining. We labelled those cases as absent pattern of nerve destruction as there were multiple granulomas leading to destruction of nerves beyond recognition. Moreover, in both of those cases, clinical presentation was highly suggestive of tuberculoid leprosy. Fleuri and
Bacchi in their study found that eight out of nine biopsies with a clinical diagnosis of tuberculoid leprosy, but with no histological or bacteriological evidence of the same, showed cutaneous nerve alteration by S-100 staining. ${ }^{5}$ Regarding the diagnostic value of $\mathrm{S}-100$ in detecting nerve components, we found the sensitivity of S-100 to be higher than that of H\&E stain in any spectra of leprosy (Table 2) which reaffirms the superiority of S-100 over H\&E stain.

In our study, fragmentation of the nerves was the predominant pattern observed in all spectra of leprosy except in tuberculoid leprosy which showed infiltrated pattern followed by absent nerve pattern as the commonest. This is in agreement with the study done by Thomas et al. where the predominant pattern of nerve damage was fragmented pattern of nerve damage followed by absent pattern of nerve damage. ${ }^{4}$ Singh et al however reported that the complete absence of nerve twigs is the most reliable criterion of leprosy. ${ }^{15}$ Skin biopsies from the 10 controls showed intact nerves only. Although few intact nerve twigs were noted focally in some of cases of leprosy, nerve fragmentation or infiltration was observed elsewhere on S-100 staining. Tirumalae et al in their study have emphasized the role of S-100 in this regard as it demonstrates both intact and normal nerves. They also noticed some of the pitfalls while interpreting S-100 staining particularly Langerhans cells, which also stain positively with S-100 and are confused with nerve cells when present within granulomas. ${ }^{12}$ In our case, we only considered S-100 stained cells as nerve cells when they were present in small clusters or along the contour of nerves.

\section{CONCLUSIONS}

S-100 immunostain is a useful auxiliary aid in the diagnosis of leprosy especially tuberculoid spectrum and intermediate leprosy. Fragmented and infiltrated patterns are the most common patterns of nerve involvement in various spectra of leprosy.

\section{ACKNOWLEDGEMENTS}

The authors would like to thank Mr. Gyanendra Sapkota and Mr. Ram Maharjan for technical support. 


\section{Conflict of Interest: None.}

\section{REFERENCES}

1. Singh A, Weng X, Nath I. Skin Biopsy in leprosy. In: Khopkar U, editor. Skin Biopsy - Perspectives. InTech;2011.p.73-86. [Full Text]

2. Mohanraj A, Srinivasan S. Role of S-100 immunostaining in demonstration of nerve changes and quantification of dendritic cells in leprosy. JCDR. 2014 Mar;8(3):38-40. [PubMed]

3. Gupta SK, Nigam S, Mandal AK, Kumar V. S-100 as a useful auxiliary diagnostic aid in tuberculoid leprosy. J Cutan Pathol. 2006; 33: 482-86. [PubMed]

4. Thomas MM, Jacob M, Chandi SM, George S, Pulimood S, Jeyaseelan L, Job CK. Role of S-100 staining in differentiating leprosy from other granulomatous disease of the skin. Int J Lepr Other Mycobact Dis. 1999 Mar; 67(1):1-5. [PubMed]

5. Fleuri RN, Bacchi CE. S-100 protein and immunoperoxidase technique as an aid in the histopathologic diagnosis of leprosy. Int J Lepr. 1987;55(2):338-44. [PubMed]

6. Ismail EA. Comparison of leprosy and Non-leprosy Granulomas by using S100 protein. JMRI 2007;28(2):181-4. Lucas S. Bacterial Diseases. In: Elder DE, editor. Lever's Histopathology of the skin. 10th ed. Wolters Kluwer; 2009.p.558-66. [Full Text]

7. Ridley DS. Histological classification and the immunological spectrum of leprosy. Bull World Health Organization. 1974;51:451. [PubMed]
8. Lowy L. Processing of biopsies for leprosy bacilli. J Med Lab Technol. 1956;13:558. [PubMed]

9. Lucas S. Bacterial Diseases. In: Elder DE, editor. Lever's Histopathology of the skin. $10^{\text {th }}$ Ed. Wolters Kluwer; 2009.p.558-66.Briton WJ, Lockwood DNJ. leprosy. Lancet. 2004;363:1201-19.

10. Noordeen SK. Eliminating leprosy as a public health problem. Int J Lepr. 1995;63:559. [PubMed]

11. Briton WJ, Lockwood DNJ. leprosy. Lancet. 2004;363:1201-19. [PubMed]

12. Tirumalae R, Stany AI, Shanubhogue S, Yeliur IK. S-100 immunostaining in the distinction of Borderline Tuberculoid leprosy from other cutaneous granulomas. Indian J Dermatol. 2014;59(4):421. [PubMed]

13. Khan AR. S-100 protein in the diagnosis of tuberculoid/ borderline tuberculoid leprosy. Ann Saudi Med. 1998;14(4):305-7. [PubMed]

14. Job CK, Drain V, Deming AT, Hastings RC, Gerber MA. Role of S-100 protein as a marker for Schwann cells in the diagnosis of tuberculoid leprosy. Int J Lepr Other Mycobact Dis. 1990;58(2):392-3. [PubMed]

15. Singh N, Arora VK, Ramam M, Tickoo SK, Bhatia A. An evaluation of S-100 stain in the histological diagnosis of Tuberculoid leprosy and other graulomatous dermatoses. Int J Lepr Other Mycobact Dis. 1994 Jun;62(2):263-7. [PubMed] 